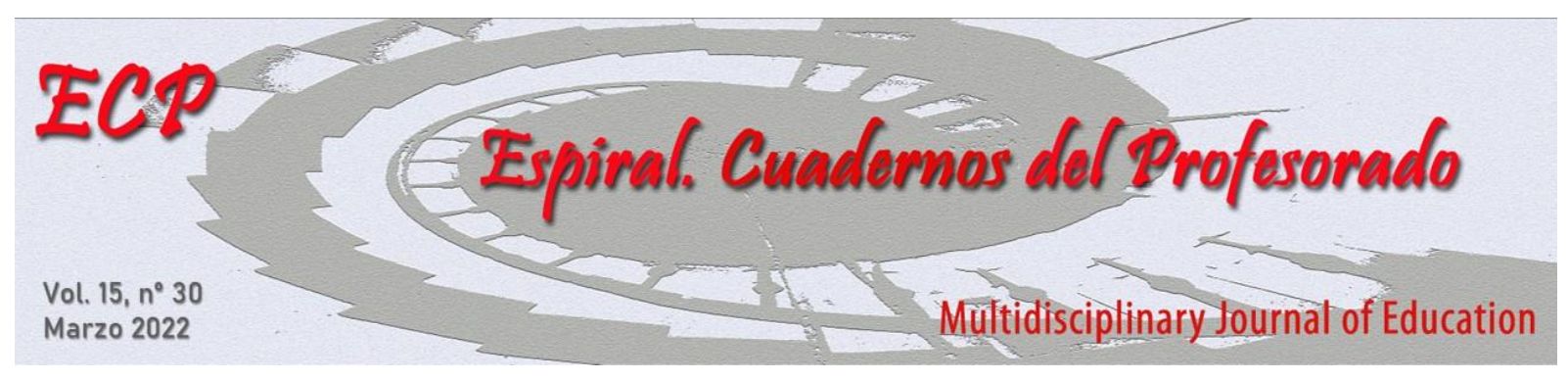

\title{
Incidencia de la Metodología JiTTwT en el rendimiento académico de estudiantes universitarios
}

\section{Incidence of JiTTwT Methodology in academic performance of university students}

\author{
Ana-Lucía Pérez-Suasnavas y Karina Cela \\ Universidad Central del Ecuador, Universidad de las Fuerzas Armadas ESPE
}

\begin{abstract}
Resumen
Con frecuencia los actores de la educación, se encuentran en la búsqueda de factores relacionados con el rendimiento académico, y que conducen al éxito o fracaso estudiantil. El objetivo del presente estudio consiste en determinar, si la participación estudiantil durante las sesiones de clases presenciales, incide en el rendimiento académico de los estudiantes universitarios, mediante la aplicación de la Metodología Justo a Tiempo para Enseñar con Twitter (JiTTwT). El método utilizado fue cuasiexperimental y transversal, con intervención de dos grupos. La muestra estuvo conformada por 144 estudiantes matriculados en los primeros niveles de la Carrera de Ingeniería Civil de una universidad pública, en el período septiembre/2019 a febrero/2020. Los hallazgos evidencian que, el rendimiento académico de los estudiantes del grupo experimental es mayor en $10.62 \%$ respecto al grupo de control, al aplicar la Metodología JiTTwT; además, el porcentaje de estudiantes del grupo experimental que aprueba la asignatura y mejora el rendimiento es mayor en $9.37 \%$, respecto del grupo de control. Con estos resultados se puede determinar que, la participación estudiantil incide en el rendimiento académico de los estudiantes.
\end{abstract}

Palabras clave: Metodología JiTTwT; estrategia de enseñanza; participación estudiantil; rendimiento académico; universidad.

\begin{abstract}
Education actors often are looking for factors related with academic performance, that determine the student's success or failure. The aim of this study is to determinate if students' participation during face-to-face class influences on the academic performance of university students by means of Just in Time to Teach with Twitter (JiTTwT) Methodology. A quasi-experimental method and cross-sectional was used with intervention of two groups. The sample was taken to 144 students signed up on first levels of Civil Engineering from a public university, during the September/2019 to February/2020 period. The results evidence students' academic performance from experimental group was $10.62 \%$ higher than control group, when it applies JiTTwT methodology. Besides, percentage from experimental group that approve the subject and increase their performance was $9.37 \%$ higher than the control group. With these results, it concludes that student's participation influence on the academic performance of university students.
\end{abstract}

Keywords: JiTTwT methodology; teaching strategies; student participation; academic performance; university.

Fecha de recepción: 22/09/2021

Fecha de aceptación: 12/11/2021

Correspondencia: Ana-Lucía Pérez-Suasnavas, Universidad Central del Ecuador Email: alperezs@uce.edu.ec 


\section{Introducción}

El rendimiento académico en la educación superior es un tema de interés a nivel internacional; tanto por el alto porcentaje de reprobación (Pineda Lezama y Alcántara Galdámez, 2017; Torres-Zapata et al., 2019; Vivas-Vivas et al., 2017); como por la calidad de estudiantes graduados (González Jaimes, 2016; Lluch Molins et al., 2017; Niama et al., 2020; Palloroso Granizo y García Rondón, 2019; Rabanal Oyarce et al., 2020); por lo que, existen estudios orientados a determinar una definición apropiada, identificar factores que inciden en el rendimiento, mejorar el desempeño o elevar el porcentaje de estudiantes aprobados (Chong González, 2017; Cortez Bailón et al., 2017).

Debido a las múltiples definiciones sobre el rendimiento académico y los factores influyentes, su conceptualización ha recibido diferentes críticas, puesto que una calificación no determina el aprendizaje alcanzado (Chilca Alva, 2017). Según Jara et al. (2008), el rendimiento académico se conoce como la capacidad de una persona para responder a estímulos, objetivos y propósitos educativos previamente establecidos; por su parte, Fernández Jiménez y Rubal Lorenzo (2014) lo definen como un indicador del nivel de aprendizaje alcanzado por el alumno (p. 2). Mientras que Garbanzo Vargas (2007) lo considera como una medida del desempeño estudiantil, de las actividades académicas desarrolladas durante un curso (p. 22).

El rendimiento académico puede verse influenciado por factores - no determinantes -, como: hábitos de estudio, autoestima, motivación; ingresos económicos, lugar de residencia; tipo de institución, horario de clase, tamaño del grupo, etc., que conducen al abandono o éxito académico (Chong González, 2017; Cortez Bailón et al., 2017; Jara et al., 2008).

Así, una participación activa durante una sesión de clase presencial, recibe influencia de agentes como: disposición, motivación, estilos de aprendizaje, relación profesor-estudiante, seguridad y confianza del estudiante, entre otros (Pérez-Suasnavas et al., 2020; Zepke y Leach, 2010). Por lo que, la participación puede ser evaluada en diferentes entornos y momentos (Bond et al., 2020; Jenaro-Río et al., 2018; Kahu, 2013; Tejera Concepción y Cardoso Sarduy, 2015).

Estudios previos, evidencian el uso de metodologías y estrategias educativas para mejorar el rendimiento académico (Fernández Solo de Zaldívar, 2017), entre ellas: el aula invertida (Mingorance et al., 2017), objetos de aprendizaje (Urbina Nájera, 2019), estrategias neuro-didácticas (Tacca Huamán et al., 2019), estrategias de búsqueda, selección y procesamiento de la información (Juárez Lugo et al., 2016), evaluación continua (García-Merino et al., 2016), uso de las TIC (Silva Quiroz y Maturana Castillo, 2017), aprendizaje colaborativo (Pimienta Prieto, 2012), redes sociales (Gallardo-López y López-Noguero, 2020), entre otras.

Sin embargo, no existen suficientes investigaciones experimentales que relacionen la participación estudiantil en las clases presenciales y el rendimiento académico en asignaturas del área de Ciencias de la Computación (Gebre et al., 2014; Griffiths et al., 2016; Payne, 2017).

El estudio realizado por Pérez-Suasnavas y Cela (2020) establece que, la aplicación de la Metodología JiTTwT a los estudiantes de la Carrera de Ingeniería Civil, mejora el nivel de participación estudiantil durante las sesiones de clases presenciales; por tanto, se pretende determinar si la participación estudiantil incide en el rendimiento académico mediante la aplicación de esta metodología.

\section{Metodología JiTTwT}

La Metodología JiTTwT, surge como una necesidad de minimizar el problema de la reducida participación de los estudiantes durante las sesiones de clases presenciales; para generar espacios de debate e intercambio de ideas, desarrollar destrezas y promover el aprendizaje significativo, mediante el uso de redes sociales y la integración de estrategias educativas adaptativas (García-Merino et al., 2016; Gómez Trigueros y Ruiz Bañuls, 2018; Silva Quiroz y Maturana Castillo, 2017). Esta metodología recientemente diseñada (Pérez-Suasnavas et al., 2020), constituye una primera parte de un proyecto de mayor alcance.

La Metodología JiTTwT está direccionada para estudiantes de una universidad pública, donde la matriculación es masiva, especialmente en los primeros niveles de formación. Por lo que, el seguimiento individual es una tarea compleja de gestionar por el docente con métodos tradicionales quien debe motivar 
continuamente a los estudiantes, en especial cuando la materia es optativa o no corresponde al eje profesionalizante de la carrera (Carlos-Martínez et al., 2018; Castillo Ramírez et al., 2013).

Investigaciones en las que se han incorporado redes sociales en la educación superior (Chávez Márquez y Gutiérrez Diez, 2015; García Suárez et al., 2015; Rodríguez Gallego et al., 2017), se evidencia la ausencia de una metodología, modelos o «buenas prácticas para su uso», según lo señala Marín-Díaz y Cabero-Almenara (2019). Las redes sociales como recursos educativos, deben acompañarse por metodologías innovadoras y adaptativas, que desarrollen destrezas y mejoren el rendimiento académico (Chávez Martínez, 2015); aspectos que la Metodología JiTTwT ha considerado, así como la opinión estudiantil (Ruiz Bolívar, 2016); motivando la participación activa, para alcanzar un aprendizaje significativo, incrementando la posibilidad de éxito como lo manifiestan Pineda Báez et al. (2014) y Urbina Nájera (2019).

\section{Secuencia Didáctica de la Metodología JiTTwT}

La metodología tiene como base la secuencia didáctica propuesta por Díaz Barriga (2006) y Feo (2010), adaptada por Pérez-Suasnavas et al. (2020) y descrita en 3 pasos: a) recolectar y analizar datos provenientes de una encuesta; b) fundamentar el diseño de la estrategia; y c) diseñar la estrategia.

Con los datos recolectados en el primer paso, se analizan e identifican los factores que inciden en el nivel de participación estudiantil durante las sesiones de clases presenciales.

En el segundo paso se establece la fundamentación de la estrategia, que incluye tanto orientaciones pedagógicas como tecnológicas.

Por último, se integran los resultados de la encuesta, con la fundamentación tecno-pedagógica, para el desarrollo de las 4 fases que incluye la metodología.

\section{Fases de la Metodología JiTTwT}

La Metodología JiTTwT contempla 4 fases que se describen a continuación:

1. Generar conocimientos previos: en esta fase se identifican los conocimientos del estudiante y se promueve nuevos saberes, mediante la recopilación de tweets sobre los aprendizajes y/o dudas de los estudiantes sobre una temática previa.

2. Orientar la atención del estudiante: se destacan los tweets más relevantes y se envía tweets de retroalimentación.

3. Organizar información: en esta fase se presenta el material didáctico que incluye un resumen de los tweets de los estudiantes. Se motiva a los estudiantes a expresar sus conclusiones o dudas que persistan. Se conforman grupos de trabajo para resolver problemas de razonamiento lógico y abstracto.

4. Enlazar conocimientos: los talleres desarrollados son evaluados entre pares. Se solicita nuevos tweets para destacar los resultados de aprendizaje y/o dudas generadas del taller, así como el envío de comentarios finales. El docente finaliza la sesión, contrastando el contenido de los tweets con el objetivo de la clase.

Con este estudio, se pretende determinar si la Metodología JiTTwT incide en el rendimiento académico de los estudiantes universitarios; para lo cual se establecieron las siguientes variables e hipótesis respectivamente:

- Variable independiente: Metodología JiTTwT.

- Variable dependiente: Rendimiento académico.

- H0: los estudiantes que reciben clases de programación utilizando la Metodología JiTTwT mejoran el rendimiento académico en la asignatura. 


\section{Método}

\section{Diseño del Estudio}

El estudio fue del tipo transversal. Para relacionar las variables, se aplicó un método cuasiexperimental por ser más aplicable al ámbito educativo, con dos grupos no equivalentes, previamente conformados: un grupo de control y un grupo experimental.

\section{Instrumento}

El instrumento se construyó a partir de los datos recolectados del registro personal del docente, correspondiente al período septiembre/2019 a febrero/2020, con información de los 144 estudiantes como las calificaciones por Hemisemestre, registro de participaciones, género, matrícula, estado final, etc.; que fueron clasificados por grupo de estudio y tabulados en hojas electrónicas. La hoja electrónica es una creación propia del autor.

Un Hemisemestre, más identificado como Hemi dentro de la Institución, corresponde a la mitad de un período académico, cuya duración es de 16 semanas; es decir, un período académico tiene 2 Hemis, cada uno de aproximadamente 2 meses.

Para validar el instrumento, se comparó el promedio de calificaciones y el estado final del estudiante, con el reporte del Sistema Integral de Información Universitaria (SIIU), del cual se obtiene, en calidad de docente, únicamente el promedio, porcentaje de asistencia y el estado del estudiante (aprobado/reprobado).

La hoja electrónica contiene los siguientes campos tabulados por grupo de estudio: código del estudiante, promedio de calificaciones por Hemi, registro de participación, estado del estudiante, análisis del rendimiento y género. Véase Figura 1.

Figura 1.

Tabulación de datos por grupo.

\begin{tabular}{|c|c|c|c|c|c|c|c|c|c|}
\hline A & B & $\mathrm{C}$ & $\mathrm{D}$ & $\mathrm{E}$ & $\mathrm{F}$ & G & $\mathrm{H}$ & 1 & $J$ \\
\hline \multicolumn{10}{|c|}{ ANÁLSIS GRUPO DE CONTROL } \\
\hline $\begin{array}{c}\text { Código } \\
\text { Estudiante }\end{array}$ & 1 Hemi & \begin{tabular}{|c|}
$\begin{array}{c}\text { Participa 1 } \\
\text { Hemi }\end{array}$ \\
\end{tabular} & 2 Hemi & \begin{tabular}{|c|}
$\begin{array}{c}\text { Participa 2 } \\
\text { Hemi }\end{array}$ \\
\end{tabular} & RETIRADO & PARTICIPA & \begin{tabular}{|c|} 
MEIORA \\
PROMEDIO \\
\end{tabular} & ESTADO & GÉNERO \\
\hline EOOO1 & 18,80 & 1 & 18,30 & 1 & NO & SI & NO & APRUEBA & $\mathrm{M}$ \\
\hline EOOO2 & 17,50 & 1 & 13,10 & 1 & NO & $\mathrm{SI}$ & NO & APRUEBA & $\mathrm{M}$ \\
\hline EOOO3 & 8,80 & 0 & 12,30 & 0 & NO & NO & $\mathrm{SI}$ & APRUEBA & $\mathrm{M}$ \\
\hline EOOO4 & 11,70 & 0 & 11,80 & 0 & NO & NO & $\mathrm{SI}$ & REPRUEBA & $\mathrm{M}$ \\
\hline E0005 & 11,20 & 1 & 1,00 & 0 & SI & $\mathrm{SI}$ & NO & REPRUEBA & $\mathrm{F}$ \\
\hline$\ldots$ & $\ldots$. & $\ldots$. & $\ldots$. & $\ldots .$. & $\ldots .$. & $\ldots$. & $\ldots$. & $\ldots .$. & $\ldots$. \\
\hline$\ldots .$. & $\ldots . .$. & $\ldots . .$. & $\ldots . .$. & $\ldots .$. & $\ldots . .$. & $\ldots . .$. & $\ldots . .$. & $\ldots . .$. & $\ldots .$. \\
\hline
\end{tabular}

De la Figura 1, se deriva la siguiente información:

- Las columnas B y D corresponden al promedio de calificación del $1^{\circ}$ y $2^{\circ}$ respectivamente. La comparación entre estos valores se refleja en la columna $\mathrm{H}$.

- Las columnas C y E registran mediante una variable dicotómica (1=si, $0=$ no) si el estudiante participó al menos una vez en cada Hemi. La comparación se registra en la columna G.

- La columna F registra si el estudiante consta como RETIRADO.

- La columna I, el estado del estudiante al finalizar el período académico.

- La columna J registra el género al que pertenece el estudiante.

- Los campos cualitativos fueron transformados a valores cuantitativos para el cálculo de las medidas de tendencia central. 


\section{Muestra}

La población estuvo conformada por estudiantes de los primeros niveles de formación $\left(2^{\circ}\right.$ y $3^{\circ}$ nivel) de una universidad pública, matriculados en el período académico septiembre/2019 a febrero/2020. Para la muestra se consideraron los estudiantes que cumplían con los siguientes criterios: 1) estudiantes de la Carrera de Ingeniería Civil; 2) estudiantes matriculados en la asignatura de Programación; 3) estudiantes que asisten regularmente a clases presenciales.

La malla curricular de Ingeniería Civil tiene dos ejes de formación: básica y profesionalizante. Programación pertenece al eje de formación básica y es obligatoria; sin embargo, es común que los estudiantes no le consideren importante dentro de su formación académica; debido a que la asignatura la cursan en los primeros niveles, y no pueden constatar su aplicabilidad en niveles superiores, donde la lógica de programación es fundamental para el desarrollo de varias aplicaciones relacionadas a la carrera.

La matriculación en una asignatura se realiza por período académico. Cada Hemi se evalúa sobre 20 puntos. Para aprobar una asignatura, se requiere la nota mínima de 28 puntos entre los 2 Hemis y registrar el $70 \%$ de asistencia.

La muestra estuvo compuesta por 144 estudiantes, cuyas edades bordeaban los 19 y 22 años, de los cuales, el $71.53 \%$ corresponden al género masculino y el $28.47 \%$ al género femenino. La muestra incluyó a los estudiantes repetidores.

Los grupos estaban previamente conformados (Hernández Sampieri et al., 2010; Murillo, 2011), y se establecieron de acuerdo a la siguiente distribución:

- Grupo de control: 64 estudiantes, con el $28.13 \%$ de género femenino y el $71.88 \%$ de género masculino. El porcentaje de repetidores fue del $7.8 \%$.

- Grupo experimental: 80 estudiantes, con el $28.75 \%$ de género femenino y el $71.25 \%$ de género masculino. El porcentaje de repetidores fue del $3.8 \%$.

\section{Resultados}

\section{Análisis Cuantitativo en el $1^{\circ} \mathrm{Hemi}$}

Con la información tabulada, se calculó la media y desviación estándar del promedio de calificaciones y de la participación estudiantil para el $1^{\circ} \mathrm{Hemi}$. Véase Tabla 1.

Tabla 1.

Medidas de tendencia central $1^{\circ}$ Hemi.

\begin{tabular}{lcccc}
\hline Grupos & \multicolumn{2}{c}{ Promedio calificaciones } \\
Media & $\begin{array}{c}\text { Desviación } \\
\text { Estándar }\end{array}$ & \multicolumn{2}{c}{$\begin{array}{c}\text { Participación estudiantil } \\
\text { Media }\end{array}$} & $\begin{array}{c}\text { Desviación } \\
\text { Estándar }\end{array}$ \\
\hline Control & 13.49 & 3.31 & .47 & .50 \\
Experimental & 13.05 & 3.11 & .33 & .47 \\
\hline
\end{tabular}

En la Tabla 1 se puede evidenciar que el grupo de control tiene una media más alta en relación al grupo experimental, tanto en calificaciones (rango de 0 a 20 puntos), como en participación.

\section{Aplicación de la Metodología JiTTwT}

La fase de experimentación con la Metodología JiTTwT, se realizó a inicios del mes de enero de 2020, que corresponde al $2^{\circ}$ Hemi, con una duración de 6 semanas. En cada sesión de clase se aplicó las 4 fases de la metodología; se utilizaron los recursos y desarrollaron las actividades descritas, de forma que se cumplieron con los objetivos propuestos.

Los estudiantes del grupo experimental eran usuarios de la red social Twitter, cuya participación fue optativa, sin generar recompensas. Por cada clase, el docente registró la participación de los dos grupos de estudio. 


\section{Análisis Cuantitativo en el $2^{\circ}$ Hemi}

Para el $2^{\circ} \mathrm{Hemi}$, se realizó el mismo cálculo que en el primero en relación a las medidas de tendencia central, con la particularidad que se incluyeron las calificaciones de los estudiantes que constaban como retirados en la asignatura. Esta consideración fue necesaria, debido a que los estudiantes que no legalizaron su retiro, constan en el sistema SIIU y sus calificaciones son consideradas para el promedio final. Véase Tabla 2.

Tabla 2.

Medidas de tendencia central $2^{\circ}$ Hemi.

\begin{tabular}{lcccccc}
\hline \multirow{2}{*}{ Grupos } & \multicolumn{2}{c}{ Promedio calificaciones } & \multicolumn{2}{c}{ Participación estudiantil } & \multicolumn{2}{c}{ Estudiantes retirados } \\
\cline { 2 - 7 } & Media & $\begin{array}{c}\text { Desviación } \\
\text { estándar }\end{array}$ & Media & $\begin{array}{c}\text { Desviación } \\
\text { estándar }\end{array}$ & Media & $\begin{array}{c}\text { Desviación } \\
\text { estándar }\end{array}$ \\
\hline Control & 12.02 & 3.48 & .44 & .50 & .02 & .13 \\
Experimental & 12.58 & 4.23 & .43 & .49 & .08 & .27 \\
\hline
\end{tabular}

En la Tabla 2 se evidencia que, la media de calificaciones en el $2^{\circ}$ Hemi es mayor en el grupo experimental al igual que los estudiantes retirados; mientras que la media de participación es mayor en el grupo de control.

\section{Análisis Cuantitativo en el $2^{\circ}$ Hemi}

Para evidenciar los cambios generados entre la participación estudiantil y el rendimiento académico entre los dos Hemis, se elaboró una tabla comparativa, con las medidas de tendencia central entre los dos grupos. Véase Tabla 3.

Tabla 3.

Comparativo de medidas de tendencia central entre el $1^{\circ}$ y $2^{\circ}$ Hemi.

\begin{tabular}{|c|c|c|c|c|}
\hline GRUPOS & \multicolumn{2}{|c|}{$1 \mathrm{Hemi}$} & \multicolumn{2}{|c|}{2 Hemi } \\
\hline DE CONTROL & Media & $\begin{array}{l}\text { Desviación } \\
\text { estándar }\end{array}$ & Media & $\begin{array}{l}\text { Desviación } \\
\text { estándar }\end{array}$ \\
\hline $\begin{array}{l}\text { Promedio de Calificaciones } \\
\text { Participación Estudiantil }\end{array}$ & $\begin{array}{c}13.49 \\
.47\end{array}$ & $\begin{array}{c}3.31 \\
.50\end{array}$ & $\begin{array}{l}12.02 \\
.44\end{array}$ & $\begin{array}{c}3.48 \\
.50\end{array}$ \\
\hline Promedio de participación & \multicolumn{2}{|c|}{$46.88 \%$} & \multicolumn{2}{|c|}{$43.75 \%$} \\
\hline EXPERIMENTAL & Media & $\begin{array}{c}\text { Desviación } \\
\text { estándar }\end{array}$ & Media & $\begin{array}{c}\text { Desviación } \\
\text { estándar }\end{array}$ \\
\hline Promedio de Calificaciones & 13.05 & 3.11 & 12.58 & 4.23 \\
\hline Participación Estudiantil & .33 & .47 & $.43(*)$ & .49 \\
\hline
\end{tabular}

En la Tabla 3, se evidencia que el promedio de calificaciones disminuyó tanto para el grupo de control como para el experimental, en 1.47 y .47 puntos respectivamente entre el $1^{\circ}$ y $2^{\circ} \mathrm{Hemi}$. Sin embargo, la participación estudiantil incrementó para el grupo experimental en el $2^{\circ} \mathrm{Hemi}$, tanto por el cálculo de la media $(*)$, como por el porcentaje registrado por el docente $(* *)$.

\section{Análisis Cualitativo al Finalizar el Curso}

Para relacionar la participación estudiantil y el rendimiento académico, se analizaron los datos por estudiante y por grupo al final del período académico, de acuerdo a los siguientes criterios:

- Porcentaje de estudiantes por grupo de estudio que participa y NO participa.

- Porcentaje de estudiantes por grupo que mejora y NO mejora su rendimiento académico entre el $1^{\circ}$ y el $2^{\circ} \mathrm{Hemi}$. 
- Porcentaje de estudiantes que aprueba o reprueba la asignatura y su rendimiento académico.

Para visualizar los datos según los criterios descritos anteriormente, se elaboró una tabla comparativa entre el grupo de control y el grupo experimental. Véase Tabla 4.

Tabla 4.

Análisis comparativo entre la participación estudiantil y el rendimiento académico.

\begin{tabular}{ccccccc}
\hline \multirow{2}{*}{ Grupo } & \multirow{2}{*}{ Participación } & \multirow{2}{*}{\begin{tabular}{c} 
Estudiantes \\
\cline { 3 - 6 }
\end{tabular}} & & \multicolumn{2}{c}{$\begin{array}{c}\text { Estudiantes mejoran } \\
\text { rendimiento }\end{array}$} & \multicolumn{2}{c}{$\begin{array}{c}\text { \% Estudiantes NO } \\
\text { mejoran rendimiento }\end{array}$} \\
\cline { 3 - 6 } De control & Participa & $60.94 \%$ & $15.63 \%$ & $.00 \%$ & $37.50 \%$ & Reprueban \\
\cline { 3 - 6 } & NO Participa & 39.06 & $6.25 \%$ & $12.50 \%$ & $10.94 \%$ & $9.37 \%$ \\
& & & & & \\
Experimental & Participa & $56.25 \%$ & $25.00 \%$ & $1.25 \%$ & $22.50 \%$ & $7.50 \%$ \\
& NO Participa & $43.75 \%$ & $10.00 \%$ & $5.00 \%$ & $13.75 \%$ & $15.00 \%$ \\
\hline
\end{tabular}

De los datos descritos en la Tabla 4 se derivan los principales resultados:

- El $60.94 \%$ de estudiantes del grupo de control que participa, es mayor que el grupo experimental $(56.25 \%)$.

- El $26.25 \%$ de estudiantes del grupo experimental que participa y mejora el rendimiento, es mayor que el grupo de control (15.63\%).

- Del $56.25 \%$ de estudiantes del grupo experimental que participa, el $25.00 \%$ participa, mejora el rendimiento y aprueba; sin embargo, este porcentaje es mayor que los del grupo de control en similares condiciones que refleja el $15.63 \%$.

- El $25.00 \%$ de estudiantes del grupo experimental que participa, mejora el rendimiento y aprueba, es mayor que el grupo de estudiantes que NO mejora el rendimiento $(22.50 \%)$.

- El $15.63 \%$ de estudiantes del grupo de control que participa, mejora el rendimiento y aprueba, es menor que el grupo de estudiantes que NO mejora el rendimiento $(37.50 \%)$.

- El $1.25 \%$ de estudiantes del grupo experimental, que mejora el rendimiento, participa y reprueba, es mayor que los del grupo de control (.00\%).

- El $6.25 \%$ de estudiantes del grupo experimental, que mejora el rendimiento y reprueba, es menor que los del grupo de control $(12.50 \%)$.

- El $5.00 \%$ de estudiantes del grupo experimental que NO participa, mejora el rendimiento y reprueba es mayor que los estudiantes que si participan $(1.25 \%)$.

- Existe un $37.50 \%$ de estudiantes que NO mejora el rendimiento académico; sin embargo, aprueba la asignatura y corresponde al grupo de control.

En razón de que los estudiantes del género masculino representan el mayor porcentaje de la muestra, no se realizó el análisis por género en relación con la participación estudiantil y el rendimiento académico.

\section{Análisis Cuantitativo Final}

Para este análisis se obtuvieron las medidas de tendencia central (media y desviación estándar) de los siguientes campos: estudiantes retirados, participación estudiantil, rendimiento académico y estudiantes que finalizan el curso, tanto los estudiantes que aprueban, como los estudiantes que reprueban.

Se consideraron los estudiantes retirados, ya que inciden en el número de estudiantes reprobados y el promedio final que consta en el sistema SIIU; como se señaló anteriormente, en el sistema institucional 
los estudiantes que no legalizaron su retiro hasta el $1^{\circ} \mathrm{Hemi}$, constan como estudiantes reprobados al final del período académico. Véase Tabla 5.

Tabla 5.

Medidas de tendencia central final.

\begin{tabular}{clcccc}
\hline Grupo & $\begin{array}{l}\text { Medida de } \\
\text { tendencia }\end{array}$ & $\begin{array}{c}\text { Estudiantes } \\
\text { retirados }\end{array}$ & $\begin{array}{c}\text { Participación } \\
\text { estudiantil }\end{array}$ & $\begin{array}{c}\text { Rendimiento } \\
\text { académico }\end{array}$ & $\begin{array}{c}\text { Estudiantes que } \\
\text { finalizan el curso }\end{array}$ \\
\hline Control & $\begin{array}{l}\text { Media } \\
\text { Desviación } \\
\text { estándar }\end{array}$ & .016 & .609 & .344 & 1.297 \\
Experimental & .125 & .492 & .479 & .460 \\
& $\begin{array}{l}\text { Media } \\
\text { Desviación } \\
\text { estándar }\end{array}$ & .075 & .563 & .413 & 1.288 \\
\hline
\end{tabular}

De la Tabla 5 se derivan los siguientes resultados:

- La media de los estudiantes retirados del grupo experimental es mayor al grupo de control.

- La media de los estudiantes que registran participación es mayor para el grupo de control.

- La media de los estudiantes que registran una variación en su rendimiento académico es mayor para el grupo experimental.

- La media de los estudiantes que finalizan el curso es mayor para el grupo de control en relación al grupo experimental.

Para relacionar la participación estudiantil y el rendimiento académico de los estudiantes, se obtuvo el coeficiente de correlación de Pearson para los dos grupos de estudio. Véase Tabla 6.

Tabla 6.

Correlación entre participación estudiantil y rendimiento académico.

\begin{tabular}{cc}
\hline Grupo & Coeficiente de Correlación \\
\hline De control & -.229677367 \\
Experimental & .124763754 \\
\hline
\end{tabular}

Los datos evidencian una correlación negativa en el grupo de control, y una correlación positiva para el grupo experimental (Hernández Lalinde et al., 2018; QuestionPro, 2017).

\section{Discusión}

Según los reportes generados del sistema SIIU entre los períodos académicos 2015 y 2019, la asignatura de Programación registra un bajo rendimiento académico, con un promedio del $36.63 \%$ de reprobación entre todos los cursos; cifras que se contrastan con los estudios realizados por Barreno-Freire et al. (2019) y Beltrán et al. (2015), quienes expresan que los estudiantes que cursan la asignatura de Programación, registran un alto porcentaje de reprobación; así como los hallazgos obtenidos del presente estudio, con un porcentaje de $39.68 \%$ de estudiantes que no mejoran el rendimiento académico, y reprueban la asignatura.

Frente a la ausencia de estudios que determinen los factores asociados al bajo rendimiento académico en la asignatura de Programación en la Carrera de Ingeniería Civil, la falta de propuestas para 
mejorar las calificaciones y minimizar la tasa de reprobación; se aplicó la Metodología JiTTwT para analizar la relación entre la participación y el rendimiento académico, y poder aceptar o rechazar la hipótesis planteada.

El grupo de control registra mayor número de estudiantes repetidores; sin embargo, debido a la falta de factores complementarios, no se puede establecer una relación entre la condición estudiantil y el rendimiento académico, como lo señala Soto-González et al. (2015) y Jenaro-Río et al. (2018), quienes manifiestan que la condición de ser estudiante repetidor no influye en el rendimiento académico y que, por el contrario, los estudiantes repetidores reflejaban una tasa menor de rendimiento.

A pesar que los grupos estaban en igualdad de condiciones al inicio del estudio, las medidas de tendencia central en el $1^{\circ} \mathrm{Hemi}$, denotan mejores valores para el grupo de control tanto en calificación como en participación estudiantil. Con estos datos, es posible establecer una relación entre la participación y el rendimiento; resultados que pueden ser comparables con los obtenidos por Aspeé et al. (2018) y Pineda Báez et al. (2014); quienes señalan que la participación activa forma parte del compromiso estudiantil y está relacionado directamente con el rendimiento académico.

Para el $2^{\circ} \mathrm{Hemi}$, el promedio de calificaciones del grupo experimental es mayor al grupo de control; lo que permite inferir que la Metodología JiTTwT genera cambios en el grupo experimental. Resultados que se pueden comparar con estudios previos (Jenaro-Río et al., 2018; Mingorance et al., 2017; Pérez Angulo y Pedroza Palomar, 2018; Urbina Nájera, 2019), donde se utiliza nuevas estrategias educativas que influyen en el rendimiento académico.

El análisis entre el $1^{\circ}$ y el $2^{\circ} \mathrm{Hemi}$, evidencia una disminución en el promedio de calificaciones en el grupo experimental, mientras que la participación estudiantil incrementó. Este resultado conduce a establecer que la Metodología JiTTwT incrementa la participación estudiantil; sin embargo, no es proporcional al rendimiento académico. En esta investigación no se analiza las causas de un bajo rendimiento, y es necesario acotar que el grupo de control también disminuyó su rendimiento en el $2^{\circ}$ Hemi.

Posterior a la aplicación de la Metodología JiTTwT, la comparación detallada de las dos variables de estudios evidencian que, el porcentaje de estudiantes del grupo experimental que registra participación, mejora su rendimiento y aprueba, es superior en $9.37 \%$ a los estudiantes del grupo de control; resultados que se pueden comparar con otros estudios previos (Campillay Briones y Meléndez Araya, 2015; GarcíaMerino et al., 2016; Griffiths et al., 2016; Jenaro-Río et al., 2018; Mingorance et al., 2017; Tacca Huamán et al., 2019; Urbina Nájera, 2019; Zepeda-Hernández et al., 2016), que destacan las ventajas del uso de nuevas metodologías, estrategias o recursos para mejorar el rendimiento académico.

Es importante destacar que, en los dos grupos de estudio existe un porcentaje considerable de estudiantes que aprobaron el curso y no mejoraron su rendimiento académico; resultado que se asemeja al realizado por Vivas-Vivas et al. (2017), quien manifiesta que los estudiantes se limitan a aprobar una asignatura con la mínima calificación y poseen un bajo interés por adquirir mayor conocimiento.

El porcentaje de estudiantes que reprueba, es mayor para el grupo de control en relación al grupo experimental; resultados que se pueden confirmar con los de Faya Hernández (2019), quien determina que los estudiantes que no son objeto de experimentación, reprueban el curso.

La inequidad de género es probable que esté relacionada a la carrera, considerada como de ciencias exactas, que registran un número reducido de estudiantes de género femenino (Arredondo Trapero et al., 2019).

Las medidas de tendencia central final indican que, la participación estudiantil del grupo de control es mayor al grupo experimental, no obstante, el grupo experimental tiene un mejor rendimiento académico. Además, el grupo de control tiene mayor cantidad de estudiantes que finalizan el curso, la causa puede estar relacionada con el número de estudiantes desertores del grupo experimental.

La correlación obtenida entre las dos variables de estudio, evidencia una correlación débil (Hernández Lalinde et al., 2018); sin embargo, el análisis completo de los resultados permite aceptar la hipótesis planteada. 
Por otra parte, es menester señalar que las limitaciones del estudio son el tamaño de la muestra, la focalización de una asignatura y la inequidad de género.

\section{Conclusiones}

Los resultados y las discusiones precedentes permiten concluir que:

En el $1^{\circ} \mathrm{Hemi}$ el grupo de control tiene un mejor rendimiento académico y mayor participación estudiantil durante las sesiones de clases presenciales. Para el $2^{\circ} \mathrm{Hemi}$, luego de la aplicación de la Metodología JiTTwT el grupo experimental registra un promedio de calificaciones mayor al grupo de control; sin embargo, la participación sigue siendo inferior al grupo de control. Se recomienda incorporar otras variables de estudio que influyan en el rendimiento académico e incrementar el tamaño de la muestra.

Los resultados del estudio demuestran que, los estudiantes del grupo experimental que aprueban la asignatura y mejoran el rendimiento académico, es mayor al porcentaje de los estudiantes del grupo de control; con estos hallazgos es posible determinar que la participación estudiantil durante las sesiones de clases presenciales, incide en el rendimiento académico de los estudiantes universitarios, mediante la aplicación de la Metodología JiTTwT.

La inclusión de nuevas metodologías o estrategias innovadoras en la educación superior, permiten mejorar el rendimiento académico de los estudiantes, cuyos resultados pueden ser evidenciados en estudios donde existe la intervención de grupos de control y experimental.

Se puede destacar que un mayor nivel de participación estudiantil dentro del aula, ya sea a través de actuaciones voluntarias o motivadas, denotan un mayor compromiso de los estudiantes y derivan un mejor rendimiento académico.

Debido a que el estudio fue del tipo transversal, y se realizó en un solo período académico, se plantea un nuevo estudio longitudinal, de forma que se pueda ratificar los resultados obtenidos.

Según el sistema SIIU, la asignatura de Programación de la Carrera de Ingeniería Civil denota un alto porcentaje de reprobación estudiantil entre los períodos 2015 y 2019; sin embargo, no existe evidencia de los factores que inciden en estos hallazgos, por lo que, se propone un tema de investigación futura que permita identificar los principales motivos, de forma que se consiga mejorar el rendimiento académico de los estudiantes y se logre minimizar el porcentaje de estudiantes reprobados.

La deserción estudiantil no forma parte del presente estudio, por lo que, se apertura un tema de investigación futura para establecer un mecanismo de seguimiento a los estudiantes, e identificar las causas probables de su deserción y tomar acciones preventivas.

La existencia de un porcentaje de estudiantes que aprueban sin mejorar el rendimiento, conduce a un análisis para determinar las causas de este resultado.

Como trabajo futuro, se requiere un análisis de las causas que generaron una disminución en las calificaciones para el $2^{\circ} \mathrm{Hemi}$ en los dos grupos de estudio.

Contribución de cada Autor: "conceptualización, A-L. P-S., K.C.; metodología, A-L. P-S.; validación, A-L. PS.; análisis, A-L. P-S.; escritura del manuscrito, A-L. P-S.; escritura, revisión y edición, A-L. P-S., K.C.; supervisión, A-L. P-S., K.C."

Financiación: Esta investigación no recibió ninguna financiación externa.

Conflicto de Intereses: Las autoras declaran que no tienen conflicto de intereses.

\section{Referencias}

Arredondo Trapero, F. G., Vázquez Parra, J. C., y Velázquez Sánchez, L. M. (2019). STEM y brecha de género en Latinoamérica. Revista de El Colegio de San Luis, 9(18), 137-158. http://dx.doi.org/10.21696/rcs19182019947

Aspeé, J. E., González, J. A., y Cavieres-Fernández, E. A. (2018). El Compromiso Estudiantil en Educación Superior como Agencia Compleja. Formación Universitaria, 11(4), 95-108. http://dx.doi.org/10.4067/S0718-50062018000400095 
Barreno-Freire, S., Haro-Jácome, O., y Flores-Yandún, P. (2019). Relación entre rendimiento académico y asistencia como factores de promoción estudiantil. Revista Cátedra, 2(1), 44-59. https://doi.org/10.29166/catedra.v2i1.1552

Beltrán, J., Sánchez, H., y Rico, M. (2015). Análisis cuantitativo y cualitativo del aprendizaje de Programación I en la Universidad Central del Ecuador. Revista Tecnológica-ESPOL, 28(5), 194-210. https://bit.ly/3vkUXxl

Bond, M., Buntins, K., Bedenlier, S., Zawacki-Richter, O., y Kerres, M. (2020). Mapping research in student engagement and educational technology in higher education: A systematic evidence map. International Journal of Educational Technology in Higher Education, 17(1), 2-30. https://doi.org/10.1186/s41239019-0176-8

Campillay Briones, S., y Meléndez Araya, N. (2015). Análisis de impacto de metodología activa y aprendizaje heurístico en asignaturas de ingeniería. Actualidades Investigativas en Educación, 15(2), 330-346. http://dx.doi.org/10.15517/aie.v15i2.18950

Carlos-Martínez, E. A., Galván Parra, L. A., Hernández Gómez, J. S., y Ruiz Moreno, R. (2018). Factores asociados al rendimiento académico en estudiantes del nivel superior pertenecientes a la etnia Yaqui. Revista de Pedagogía Crítica, 2(6), 1-8. https://bit.ly/3aBr03Q

Castillo Ramírez, A., Izar Landeta, J. M., y Espericueta González, D. E. (2013). Correlación entre trayectoria académica y el examen nacional de egreso de la licenciatura. Revista de Investigación Educativa, O(17), 172-188. https://doi.org/10.25009/cpue.v0i17.427

Chávez Márquez, I. L., y Gutiérrez Diez, M. del C. (2015). Redes sociales como facilitadoras del aprendizaje de ciencias exactas en la educación superior. Revista Apertura, 7(2), 49-61. https://bit.ly/3erngmN

Chávez Martínez, J. de J. (2015). Uso de las redes educativas en la educación superior. Un caso específico. Revista Científica de Comunicación, 6(1), 82-96. https://bit.ly/3vjGv8v

Chilca Alva, M. L. (2017). Autoestima, hábitos de estudio y rendimiento académico en estudiantes universitarios. Propósitos y Representaciones, 5(1), 71-127. https://doi.org/10.20511/pyr2017.v5n1.145

Chong González, E. G. (2017). Factores que inciden en el rendimiento académico de los estudiantes de la Universidad Politécnica del Valle de Toluca. Revista Latinoamericana de Estudios Educativos (México), 47(1), 91-108. https://doi.org/10.48102/rlee.2017.47.1.159

Cortez Bailón, F. M., Tutiven Campos, J. L., y Villavicencio Morejón, M. N. (2017). Determinantes del Rendimiento Académico Universitario. Revista Publicando, 4(10 (1)), 284-296. https://bit.ly/3nASmvh

Díaz Barriga, Á. (2006). El enfoque de competencias en la educación: ¿Una alternativa o un disfraz de cambio? Perfiles Educativos, 28(111), 7-36. https://bit.ly/3nkGTBe

Faya Hernández, M. (2019). Relación de la metodología universitaria con la reprobación de asignaturas de ingeniería civil de la Universidad Nacional de Cajamarca-sección Jaén, periodo 2007-2010 [Universidad Nacional de Cajamarca]. https://bit.ly/3pGkGOx

Feo, R. (2010). Orientaciones básicas para el diseño de estrategias didácticas. Tendencias Pedagógicas, 16, 221236. https://bit.ly/2RevMuK

Fernández Jiménez, M. E., y Rubal Lorenzo, N. (2014). Los hábitos de estudio y el rendimiento académico en el primer año de la Carrera de Medicina. Morfovirtual 2014, 1-13. https://bit.ly/2UK42PM

Fernández Solo de Zaldívar, I. (2017). Mejora de competencias: Introducción de la gestión de calidad en nuevas metodologías educativas. Profesorado. Revista de Currículum y Formación de Profesorado, 21(2), 279308. https://bit.ly/3pNbDf0

Gallardo-López, J. A., y López-Noguero, F. (2020). Twitter como recurso metodológico en Educación Superior: Una experiencia educativa con estudiantes de Trabajo Social. ALTERIDAD. Revista de Educación, 15(2), 174-189. https://doi.org/10.17163/alt.v15n2.2020.03

Garbanzo Vargas, G. M. (2007). Factores asociados al rendimiento académico en estudiantes universitarios, una reflexión desde la calidad de la educación superior pública. Revista Educación, 31(1), 43-63. https://doi.org/10.15517/revedu.v31i1.1252

García Suárez, J., Trigueros Cervantes, C., y Rivera García, E. (2015). Twitter as a resource to evaluate the university teaching process. RUSC: Revista de Universidad y Sociedad del Conocimiento, 12(3), 32-45. http://dx.doi.org/10.7238/rusc.v12i3.2092 
García-Merino, J. D., Urionabarrenetxea, S., y Bañales-Mallo, A. (2016). Cambios en metodologías docentes y de evaluación: ¿Mejoran el rendimiento del alumnado universitario? Revista Electrónica de Investigación Educativa, 18(3), 1-18. https://bit.ly/31NblCl

Gebre, E., Saroyan, A., y Bracewell, R. (2014). Students' engagement in technology rich classrooms and its relationship to professors' conceptions of effective teaching. British Journal of Educational Technology, 45(1), 83-96. https://doi.org/10.1111/bjet.12001

Gómez Trigueros, I. M., y Ruiz Bañuls, M. (2018). Interdisciplinariedad y TIC: nuevas metodologías docentes aplicadas a la enseñanza superior. Píxel-Bit. Revista de Medios y Educación, O(52). https://doi.org/10.12795/pixelbit.2018.i52.05

González Jaimes, E. I. (2016). Competencias académicas de los egresados universitarios y su predicción de ocupación laboral. RICSH Revista Iberoamericana de las Ciencias Sociales y Humanísticas, 5(10). https://bit.ly/32VO4qb

Griffiths, L., Villarroel, R., y Ibacache, D. (2016). Implementación del modelo de aula Invertida para el aprendizaje activo de la programación en ingeniería. XXIX Congreso Chileno de Educación en Ingeniería. https://bit.ly/2VqBqvi

Hernández Lalinde, J. D., Espinosa Castro, J. F., Peñaloza Tarazona, M. E., Rodriguez, J. E., Chacón Rangel, J. G., Toloza Sierra, C. A., Arenas Torrado, M. K., Carrillo Sierra, S. M., y Bermúdez Pirela, V. J. (2018). Sobre el uso adecuado del coeficiente de correlación de Pearson: Definición, propiedades y suposiciones. Archivos Venezolanos de Farmacología y Terapéutica, 35(5). https://bit.ly/3nkqexx

Hernández Sampieri, R., Fernández Collado, C., y Baptista Lucio, M. del P. (2010). Metodología de la investigación ( $5^{\mathrm{a}}$ ed.). McGraw-Hill. http://bit.ly/385JdT8

Jara, D., Velarde, H., Gordillo, G., Guerra, G., León, I., Arroyo, C., y Figueroa, M. (2008). Factores influyentes en el rendimiento académico de estudiantes del primer año de medicina. Anales de la Facultad de Medicina, 69(3), 193-197. https://doi.org/10.15381/anales.v69i3.1140

Jenaro-Río, C., Castaño-Calle, R., Martín-Pastor, M. E., y Flores-Robaina, N. (2018). Rendimiento académico en educación superior y su asociación con la participación activa en la plataforma Moodle. Estudios sobre Educación, 34, 177-198. https://doi.org/10.15581/004.34.177-198

Juárez Lugo, C. S., Rodríguez Hernández, G., Escoto Ponce de León, M. del C., y Luna Montijo, E. (2016). Relación de los estilos y estrategias de aprendizaje con el rendimiento académico en estudiantes universitarios. Revista de Estilos de Aprendizaje, 9(17). https://bit.ly/2IUGuFw

Kahu, E. R. (2013). Framing student engagement in higher education. Studies in Higher Education, 38(5), 758773. https://doi.org/10.1080/03075079.2011.598505

Lluch Molins, L., Fernández-Ferrer, M., Pons Seguí, L., y Cano García, E. (2017). Competencias profesionales de los egresados universitarios: Estudio de casos en cuatro titulaciones. Revista Qurriculum, 30, 49-64. https://bit.ly/2Hh5JRH

Marín-Díaz, V., y Cabero-Almenara, J. (2019). Las redes sociales en educación: Desde la innovación a la investigación educativa. Revista Iberoamericana de Educación a Distancia, 22(2), 25-33. https://doi.org/10.5944/ried.22.2.24248

Mingorance, A. C., Trujillo, J. M., Cáceres, P., y Torres, C. (2017). Mejora del rendimiento académico a través de la metodología de aula invertida centrada en el aprendizaje activo del estudiante universitario deficiencias de la educación. Journal of Sport and Health Research, 9(1), 129-136. https://bit.ly/3lLLYRb

Murillo, J. (2011). Métodos de investigación de enfoque experimental. https://bit.ly/2VqrNgg

Niama, L. M., Villalva, C. I., Rodríguez, Á. E., y Robalino, M. S. (2020). La competitividad y otros factores que influyen en la inserción laboral: Caso de los egresados en ingeniería de sistemas de ESPOCH, Ecuador. Revista ESPACIOS, 41(39), 50-58. https://bit.ly/38VQE31

Palloroso Granizo, R. Y., y García Rondón, I. (2019). Seguimiento a graduados: Importancia y principales experiencias internacionales. Análisis del tópico en la República del Ecuador. Caribeña de Ciencias Sociales, julio. https://bit.ly/3fgNOXL

Payne, L. (2017). Student engagement: Three models for its investigation. Journal of Further and Higher Education, 43(5), 641-657. https://doi.org/10.1080/0309877X.2017.1391186

Pérez Angulo, J. A., y Pedroza Palomar, O. V. (2018). LM1: Una metodología de estudio para la asignatura "Programación 1". Educere: Revista Venezolana de Educación, 73, 635-648. https://bit.ly/3luOk6f 
Pérez-Suasnavas, A.-L., y Cela, K. (2020). Aplicación de la Metodología JiTTwT y su incidencia en las aulas universitarias. En G. Gómez García, M. Ramos Navas-Parejo, C. Rodríguez Jiménez, y J. C. de la Cruz Campos (Eds.), Teoría y práctica en investigación educativa: Una perspectiva internacional (pp. 13431356). Dykinson S. L. https://bit.ly/3xnXEQe

Pérez-Suasnavas, A.-L., Cela, K., y Hasperué, W. (2020). Propuesta de Estrategia Educativa, para Fomentar la Participación Estudiantil Universitaria. En E. Archundia Sierra, M. Á. León Chávez, y C. Cerón Garnica (Eds.), Redes de aprendizaje digital en nodos colaborativos (pp. 240-261). Benemérita Universidad Autónoma de Puebla. https://bit.ly/3xmD82M

Pimienta Prieto, J. (2012). Estrategias de enseñanza-aprendizaje. Docencia universitaria basada en competencias (M. Vega, Ed.; Primera). Pearson. https://bit.ly/2xBDhoj

Pineda Báez, C., Bermúdez Aponte, J. J., Rubiano Bello, Á., y Pava García, N. (2014). Compromiso Estudiantil y Desempeño Académico en el Contexto Universitario Colombiano. Revista Electrónica de Investigación y Evaluación Educativa, 20(2), 1-20. https://doi.org/10.7203/relieve.20.2.4238

Pineda Lezama, O. B., y Alcántara Galdámez, N. J. (2017). Hábitos de estudio y rendimiento académico en estudiantes universitarios. Innovare: Revista de Ciencia y Tecnología, 6(2), 19-34. https://doi.org/10.5377/innovare.v6i2.5569

QuestionPro. (2017). ¿Existen diferencias entre cuestionarios y encuestas? QuestionPro. https://www.questionpro.com/blog/es/diferencias-entre-cuestionarios-y-encuestas/

Rabanal Oyarce, R., Huamán Muñoz, C. R., Murga Valderrama, N. L., y Chauca Valqui, P. (2020). Desarrollo de competencias personales y sociales para la inserción laboral de egresados universitarios. Revista de Ciencias Sociales, 26(2), 250-258. http://dx.doi.org/10.31876/rcs.v26i2.32438

Rodríguez Gallego, M. R., López Martínez, A., y Martín Herrera, I. (2017). Percepciones de los estudiantes de Ciencias de la Educación sobre las redes sociales como metodología didáctica. Pixel-Bit. Revista de Medios y Educación, 50, 77-93. http://dx.doi.org/10.12795/pixelbit.2016.i50.05

Ruiz Bolívar, C. (2016). Redes Sociales y Educación Universitaria. Paradigma, 37(1), 232-256. https://bit.ly/3vi3iBG

Silva Quiroz, J., y Maturana Castillo, D. (2017). Una propuesta de modelo para introducir metodologías activas en educación superior. Innovación educativa (México, DF), 17(73), 117-131. https://bit.ly/3pGRdE9

Soto-González, M., Cuña-Carrera, I. D., Lantarón-Caeiro, E. M., y Labajos-Manzanares, M. T. (2015). Influencia de las variables sociodemográficas y socioeducativas en el rendimiento académico de alumnos del Grado en Fisioterapia. Revista de la Fundación Educación Médica, 18(6), 397-404. https://doi.org/10.33588/fem.186.813

Tacca Huamán, D. R., Tacca Huamán, A. L., y Alva Rodriguez, M. A. (2019). Estrategias neurodidácticas, satisfacción y rendimiento académico en estudiantes universitarios. Cuadernos de Investigación Educativa, 10(2), 15-32. https://doi.org/10.18861/cied.2019.10.2.2905

Tejera Concepción, J. F., y Cardoso Sarduy, M. A. (2015). Tratamiento de las habilidades comunicativas en el contexto universitario. Revista Universidad y Sociedad, 7(2), 168-172. http://bit.ly/32tZccA

Torres-Zapata, Á. E., Acuña-Lara, J. P., y Moguel Ceballos, J. E. (2019). Índice de reprobación en universitarios. Una aproximación para la toma de decisiones. Mikarimin. Revista Científica Multidisciplinaria., 5(2), 6166. https://bit.ly/3nFEC2o

Urbina Nájera, A. B. (2019). Estrategia tecnológica para mejorar el rendimiento académico universitario. PixelBit: Revista de Medios y Educación, 56, 71-93. https://doi.org/10.12795/pixelbit.2019.i56.04

Vivas-Vivas, R., Lastra-Bravo, X., y Yépez, B. (2017). Enfoques de aprendizaje y rendimiento académico universitario en ecuador. Revista de Investigación Enlace Universitario, 16(1), 23-30. https://bit.ly/32Z0EFq

Zepeda-Hernández, S., Mena, R. A., y Ornelas, E. L. (2016). Integración de gamificación y aprendizaje activo en el aula. Revista Científica Ra-Ximhai, 12(6), 315-325. https://doi.org/10.35197/rx.12.01.e3.2016.21.sz

Zepke, N., y Leach, L. (2010). Improving student engagement: Ten proposals for action. Active Learning in Higher Education, 11(3), 167-177. https://doi.org/10.1177/1469787410379680 